Research Paper

\title{
Near Infrared Photoimmunotherapy in the Treatment of Pleural Disseminated NSCLC: Preclinical Experience
}

\author{
Kazuhide Sato, Tadanobu Nagaya, Peter L. Choyke, and Hisataka Kobayashi ${ }^{\bowtie}$ \\ Molecular Imaging Program, Center for Cancer Research, National Cancer Institute, Bethesda, MD 20892-1088. \\ $\triangle$ Corresponding author: Hisataka Kobayashi, MD. PhD. Molecular Imaging Program, Center for Cancer Research, National Cancer Institute, NIH, \\ Building 10, RoomB3B69, MSC1088, Bethesda, MD 20892-1088. Phone: 301-435-4086, Fax: 301-402-3191. E-mail: Kobayash@mail.nih.gov. \\ () 2015 Ivyspring International Publisher. Reproduction is permitted for personal, noncommercial use, provided that the article is in whole, unmodified, and properly cited. \\ See http://ivyspring.com/terms for terms and conditions.
}

Received: 2015.01.10; Accepted: 2015.02.23; Published: 2015.03.19

\begin{abstract}
Pleural metastases are common in patients with advanced thoracic cancers and are a cause of considerable morbidity and mortality yet is difficult to treat. Near Infrared Photoimmunotherapy (NIR-PIT) is a cancer treatment that combines the specificity of intravenously injected antibodies for targeting tumors with the toxicity induced by photosensitizers after exposure to NIR-light. Herein, we evaluate the efficacy of NIR-PIT in a mouse model of pleural disseminated non-small cell lung carcinoma (NSCLC). In vitro and in vivo experiments were conducted with a HER2, luciferase and GFP expressing NSCLC cell line (Calu3-luc-GFP). An antibody-photosensitizer conjugate (APC) consisting of trastuzumab and a phthalocyanine dye, IRDye-700DX, was synthesized. In vitro NIR-PIT cytotoxicity was assessed with dead staining, luciferase activity, and GFP fluorescence intensity. In vivo NIR-PIT was performed in mice with tumors implanted intrathoracic cavity or in the flank, and assessed by tumor volume and/or bioluminescence and fluorescence thoracoscopy. In vitro NIR-PIT-induced cytotoxicity was light dose dependent. In vivo NIR-PIT led significant reductions in both tumor volume $(p=0.002$ vs. APC) and luciferase activity $(p=0.0004$ vs. APC) in a flank model, and prolonged survival $(P<0.0001)$. Bioluminescence indicated that NIR-PIT lead to significant reduction in pleural dissemination ( 1 day after PIT; $p=0.0180$ ). Fluorescence thoracoscopy confirmed the NIR-PIT effect on disseminated pleural disease. In conclusion, NIR-PIT has the ability to effectively treat pleural metastases caused by NSCLC in mice. Thus, NIR-PIT is a promising therapy for pleural disseminated tumors.
\end{abstract}

Key words: photoimmunotherapy, fluorescence thoracoscopy, pleural dissemination, bioluminescence imaging, fluorescence imaging.

\section{Introduction}

Lung cancer is the most common cause of cancer-related deaths worldwide. In the USA in 2014, 224,210 people were diagnosed with lung cancer and 159,260 died [1]. Lung cancer is an aggressive disease with a very low 5 -year survival. About $80 \%$ of lung cancers are histologically classified as non-small cell lung carcinoma (NSCLC). During the course of lung cancer, pleural spread of NSCLC, which is a lethal complication, frequently occurs in advanced patients [2]. Although early stage and locally advanced NSCLC can be treated with a combination of surgery, chemotherapy, and radiation therapy, palliative chemotherapy is the only practical treatment for NSCLC with pleural metastases, resulting in only 6-9 month median survival [3]. In recognition of the poor prognosis associated with pleural metastasis, such disease has recently been reclassified from T4 to M1a [4]. Therefore, therapies that could treat pleural metastases without excessive collateral damage to the lungs might be predicted to prolong survival.

Intrapleural conventional photodynamic therapy (PDT) has been previously tested in patients after surgical debulking of pleural disease [5]. However, this treatment (using porfimer sodium as the PDT 
agent) produced some toxicities due to the poor selectivity of the agent. PDT for malignant pleural mesothelioma was also performed after surgical debulking and immunochemotherapy, this phase III study for malignant pleural mesothelioma failed to show a difference in overall survival or progression free survival for the group with additional intraoperative PDT [6]. More recently, a phase II trial of pleural PDT after surgery for NSCLC with pleural spread demonstrated that surgery and conventional PDT could be performed safely resulting in good local control and prolonged median survival [7] Thus, conventional PDT results in equivocal benefits for patients with metastases to the pleural. One clear problem with conventional PDT is that produces considerable damage to adjacent tissues thus, negating any potential benefit from the treatment itself.

The concept of using targeted light therapy is over three decades old [8,9]. However, the original PDT agents were highly hydrophobic and therefore the pharmacokinetics of antibody conjugated PDT agents were difficult to target to tumors alone. Previous studies have attempted to target conventional PDT agents by conjugating them to antibodies. Unfortunately, these conjugates were usually trapped in the liver and could only be used in isolated body cavities such as the peritoneum $[10,11]$. A study using a more hydrophilic phthalocyanine-based photosensitizer (Aluminum (III) Phthalocyanine Tetrasulfonate) has been published, however, no in vivo treatment response data was reported [12]. The recognition that substituting a water soluble phthalocyanine-based photosensitizer (IR700) in the conjugation with an antibody and applying near infrared light has led to much higher selectivity. NIR-PIT differs from these prior PDT not only in the water-solubility of the photosensitizer, but also in its reliance on NIR light that has better tissue penetration than the lower wavelengths used for exciting PDT agents. This antibody-photosensitizer conjugates (APC) demonstrates similar intravenous pharmacokinetics to naked antibodies, resulting in highly targeted tumor accumulation with minimal non-target binding. When bound to targeted cells, APCs induce rapid, selective cytotoxicity after exposure to NIR light. In vitro studies have demonstrated that NIR-PIT is highly target cell-specific and leads to rapid and irreversible cell death due to membrane damage [13-16].

One obvious limitation of NIR-PIT is that it would seem limited to tumors located relatively shallow from the surface that can be easily exposed to NIR light. However, light can be administered endoscopically and among the organs, the lungs have the best ability to transmit light because they are mostly filled with air. Thus, NIR light administered to the thoracic cavity could easily penetrate within pleural disease. In this study, we investigate the efficacy of NIR-PIT for treating pleural disease in a NSCLC mouse model.

\section{Materials and methods}

\section{Reagents}

Water soluble, silicon-phthalocyanine derivative, IRDye 700DX NHS ester and IRDye 800CW NHS ester were obtained from LI-COR Bioscience (Lincoln, NE, USA). Panitumumab, a fully humanized $\operatorname{IgG}_{2}$ $\mathrm{mAb}$ directed against EGFR, was purchased from Amgen (Thousand Oaks, CA, USA). Trastuzumab, 95\% humanized $\operatorname{IgG}_{1} \mathrm{mAb}$ directed against HER2, was purchased from Genentech (South San Francisco, CA, USA). All other chemicals were of reagent grade.

\section{Synthesis of Antibody-dye conjugates}

Conjugation of dyes with mAbs was performed according to previous reports $[13,14]$. In brief, panitumumab or trastuzumab (1 mg, $6.8 \mathrm{nmol})$ was incubated with IR700 NHS ester $(60.2 \mu \mathrm{g}, 30.8 \mathrm{nmol})$ or IR800CW NHS ester $(35.9 \mu \mathrm{g}, 30.8 \mathrm{nmol})$ in $0.1 \mathrm{~mol} / \mathrm{L}$ $\mathrm{Na}_{2} \mathrm{HPO}_{4}(\mathrm{pH}$ 8.6) at room temperature for $1 \mathrm{hr}$. The mixture was purified with a Sephadex G25 column (PD-10; GE Healthcare, Piscataway, NJ, USA). The protein concentration was determined with Coomassie Plus protein assay kit (Thermo Fisher Scientific Inc, Rockford, IL, USA) by measuring the absorption at $595 \mathrm{~nm}$ with spectroscopy (8453 Value System; Agilent Technologies, Santa Clara, CA, USA). The concentration of IR700 or IR800 was measured by absorption at $689 \mathrm{~nm}$ or $774 \mathrm{~nm}$ respectively to confirm the number of fluorophore molecules conjugated to each $\mathrm{mAb}$. The synthesis was controlled so that an average of four IR700 molecules or two IR800 molecules were bound to a single antibody. We performed SDS-PAGE as a quality control for each conjugate as previously reported [13]. We abbreviate IR700 conjugated to trastuzumab as tra-IR700, to panitumumab as pan-IR700 and IR800 conjugated to trastuzumab as tra-IR800.

\section{Cell culture}

HER2 and luciferase/GFP-expressing Calu3-luc-GFP cells were established with a transfection of RediFect Red-FLuc-GFP (PerkinElmer, Waltham, MA, USA). High GFP and luciferase expression was confirmed with 10 passages of the cells. Balb/3T3 cells were transfected with RFP (EF1a)-Puro lentiviral particles (AMSBIO, Cambridge, MA, USA). High, stable RFP expression was confirmed after 10 passages in the absence of a selection agent. To evaluate specific cell killing by NIR-PIT, 3T3 cells stably expressing RFP (3T3-RFP) were used as negative controls. Cells were 
grown in RPMI 1640 (Life Technologies, Gaithersburg, MD, USA) supplemented with $10 \%$ fetal bovine serum and $1 \%$ penicillin/streptomycin (Life Technologies) in tissue culture flasks in a humidified incubator at $37^{\circ} \mathrm{C}$ at an atmosphere of $95 \%$ air and $5 \%$ carbon dioxide.

\section{Flow Cytometry}

Fluorescence from cells after incubation with pan-IR700 or tra-IR700 was measured with a flow cytometer (FACS Calibur, BD BioSciences, San Jose, CA, USA) and CellQuest software (BD BioSciences). Calu3-luc-GFP cells $\left(1 \times 10^{5}\right)$ were incubated with each $\mathrm{APC}$ for $6 \mathrm{hr}$ at $37^{\circ} \mathrm{C}$. To validate the specific binding of the conjugated antibody, excess antibody $(50 \mu \mathrm{g})$ was used to block $0.5 \mu \mathrm{g}$ of antibody-dye conjugates [16].

\section{Fluorescence microscopy}

To detect the antigen specific localization of antibody-dye conjugates, fluorescence microscopy was performed (IX61 or IX81; Olympus America, Melville, NY, USA). Ten thousand cells were seeded on cover-glass-bottomed dishes and incubated for $24 \mathrm{hr}$. Tra-IR700 was then added to the culture medium at 10 $\mu \mathrm{g} / \mathrm{mL}$ and incubated at $37^{\circ} \mathrm{C}$ for $6 \mathrm{hr}$. The cells were then washed with PBS; Propidium Iodide (PI)(1:2000)(Life Technologies) and Cytox Blue (1:500)(Life Technologies), were used to detect dead cells. They were added into the media $30 \mathrm{~min}$ before the observation. The cells were then exposed to NIR light $\left(2 \mathrm{~J} / \mathrm{cm}^{2}\right)$ and serial images were obtained. The filter was set to detect IR700 fluorescence with a $590-650 \mathrm{~nm}$ excitation filter, and a $665-740 \mathrm{~nm}$ band pass emission filter.

Analysis of the images was performed with ImageJ software (http://rsb.info.nih.gov/ij/) [17].

\section{In vitro NIR-PIT}

One hundred thousand cells were seeded into 24 well plates or ten million cells were seeded onto a 10 $\mathrm{cm}$ dish and incubated for $24 \mathrm{hr}$. Medium was replaced with fresh culture medium containing 10 $\mu \mathrm{g} / \mathrm{mL}$ of tra-IR700 which was incubated for $6 \mathrm{hr}$ at $37^{\circ} \mathrm{C}$. After washing with PBS, phenol red free culture medium was added. Then, cells were irradiated with a NIR laser, which emits light at 685 to $695 \mathrm{~nm}$ wavelength (BWF5-690-8-600-0.37; B\&W TEK INC., Newark, DE, USA). The actual power density of $\mathrm{mW} / \mathrm{cm}^{2}$ was measured with an optical power meter (PM 100, Thorlabs, Newton, NJ, USA).

\section{Cytotoxicityl Phototoxicity assay}

The cytotoxic effects of NIR-PIT with tra-IR700 were determined by the luciferase activity and flow cytometric PI staining. For luciferase activity, 150 $\mu \mathrm{g} / \mathrm{mL}$ of D-luciferin-containing media (Gold Biotechnology, St Louis, MO, USA) was administered to PBS-washed cells $1 \mathrm{hr}$ after NIR-PIT, and analyzed on a bioluminescence imaging (BLI) system (Photon Imager; Biospace Lab, Paris, France). For the flow cytometric assay, cells were trypsinized $1 \mathrm{hr}$ after treatment and washed with PBS. PI was added to the cell suspension (final $2 \mu \mathrm{g} / \mathrm{mL}$ ) and incubated at room temperature for $30 \mathrm{~min}$, prior to flow cytometry.

To investigate the specificity of tra-IR700, excess trastuzumab $1,000 \mu \mathrm{g} / \mathrm{mL}$ added to the medium for 1 $\mathrm{hr}$, and $10 \mu \mathrm{g} / \mathrm{mL}$ of tra-IR700 was added to the media for $6 \mathrm{hr}$. Without washing with PBS, NIR light was administered and $1 \mathrm{hr}$ later PI staining was performed as above.

\section{Estimation of GFP fluorescence intensity in vitro}

Two hundred thousand cells were seeded on cover-glass-bottomed dishes and incubated for $12 \mathrm{hr}$. Tra-IR700 was then added to the culture medium (phenol red free) at $10 \mu \mathrm{g} / \mathrm{mL}$ and incubated at $37^{\circ} \mathrm{C}$ for $6 \mathrm{hr}$, followed by NIR-PIT. Cells were trypsinized $1 \mathrm{hr}$ after treatment and washed with PBS, then analyzed by flow cytometry.

\section{Animal and tumor models}

All in vivo procedures were conducted in compliance with the Guide for the Care and Use of Laboratory Animal Resources (1996), US National Research Council, and approved by the local Animal Care and Use Committee. Six- to eight-week-old female homozygote athymic nude mice were purchased from Charles River (NCI-Frederick). During procedures, the mice were anesthetized with inhaled isoflurane.

Six million Calu3-luc-GFP cells were injected subcutaneously in the right dorsum of the mice. The greatest longitudinal diameter (length) and the greatest transverse diameter (width) were measured with an external caliper. Tumor volumes based on caliper measurements were calculated by the following formula; tumor volume $=$ length $\times$ width $^{2} \times 0.5$. Tumors reaching approximately $100 \mathrm{~mm}^{3}$ in volume were selected for further experiments. Body weight was checked on the scale.

For BLI, D-luciferin $(15 \mathrm{mg} / \mathrm{mL}, 200 \mu \mathrm{L})$ was injected intraperitoneally and the mice were analyzed with a Photon Imager for luciferase activity at day 11. Mice were selected for further study based on tumor size and bioluminescence.

In order to create a pleural disseminated NSCLC model, six million Calu3-luc-GFP NSCLC cells in PBS (total $200 \mu \mathrm{L}$ ) were injected into the thoracic cavity through a right intercostal space using a 30G needle. 
To avoid lung injury, the needle could only be inserted $5 \mathrm{~mm}$ (a foam styrol stopper prevented deeper insertion). Twenty days later, bioluminescence was performed after D-luciferin $(15 \mathrm{mg} / \mathrm{mL}, 200 \mu \mathrm{L})$ was injected intraperitoneally and the mice were imaged with the Photon Imager; mice with sufficient activity were selected for further study.

\section{In vivo fluorescence imaging}

In vivo fluorescence images were obtained with a Pearl Imager (LI-COR Bioscience) for detecting IR700/ IR800 fluorescence, and a Maestro Imager (CRi, Woburn, MA, USA) for GFP. For GFP, a band-pass filter from 445 to $490 \mathrm{~nm}$ (excitation) and a long-pass blue filter over $515 \mathrm{~nm}$ (emission) were used. The tunable emission filter was automatically stepped in $10 \mathrm{~nm}$ increments from 500 to $600 \mathrm{~nm}$ for the green filter sets at a constant exposure (1000 $\mathrm{msec}$ ). The spectral fluorescence images consist of autofluorescence spectra and the spectra from GFP (Calu3-luc-GFP tumor), which were then unmixed, based on the characteristic spectral pattern of GFP, using Maestro software (CRi).

\section{Fluorescence thoracoscopy}

A model BF XP-60 bronchoscope system was inserted by a trained bronchoscopist/thoracosciopist (KS) via an intercostal space after the animal was euthanized, and the intrathoracic cavity was observed with white light and fluorescence imaging using multi-band excitation filters. Thoracoscopic images were obtained via a dichroic splitter, in which both the excitation light images were displayed using the image processor (OTV-S7; Olympus Co., Tokyo, Japan; not commercially available), and the fluorescence images, which were filtered by in-house designed multicolor emission filters ( 516 to $556 \mathrm{~nm}$ band-pass for GFP and 680 to $710 \mathrm{~nm}$ band-pass for IR700) were detected with an (EM)-CCD camera (Texas Instruments, Dallas, TX, USA). Both images were displayed side by side on the PC monitor with DualView 2 software (RGB Spectrum). Real-time images of both white light and fluorescence images were recorded. Camera gain, exposure time, and binning for the fluorescence images were held constant in each fluorescent protein throughout the study. Analysis of the images was performed with ImageJ software (http://rsb.info. nih.gov/ij/).

\section{Characterization of the pleural disseminated mouse model}

Both the disseminated pleural model and the subcutaneous bilateral flank models received $100 \mu \mathrm{g}$ of tra-IR700 or tra-IR800 intravenously (tra-IR800 was used to avoid auto-fluorescence). One day after injection, serial images were performed with a fluores- cence imager (Pearl Imager) for detecting IR700/ IR800 fluorescence, with the Photon Imager for BLI, and the Olympus BF XP-60 thoracoscopy. Images of the mice were obtained with an iphone5 (Apple Inc., Cupertino, CA, USA).

\section{In vivo NIR-PIT}

Calu3-luc-GFP right dorsum tumor xenografts were randomized into 4 groups of at least 7 animals per group undergoing one of the following treatments: (repeated PIT)[18]: (1) no treatment (control); (2) only NIR light exposure at $50 \mathrm{~J} / \mathrm{cm}^{2}$ on day 1 and $100 \mathrm{~J} / \mathrm{cm}^{2}$ on day 2; (3) $100 \mu \mathrm{g}$ of tra-IR700 i.v., no NIR light exposure; (4) $100 \mu \mathrm{g}$ of tra-IR700 i.v., NIR light was administered at $50 \mathrm{~J} / \mathrm{cm}^{2}$ on day 1 after injection and $100 \mathrm{~J} / \mathrm{cm}^{2}$ on day 2 after injection. These therapies were performed only once at day 14 after cell implantation. Mice were monitored daily, and tumor volumes and body weight were measured three times a week until the tumor diameter reached $2 \mathrm{~cm}$, whereupon the mouse was euthanized with carbon dioxide.

In vivo imaging was acquired with a fluorescence imager (Pearl Imager) for detecting IR700 fluorescence, and the Photon Imager for BLI. For analyzing BLI, ROI of similar size were placed over the entire tumor.

For evaluation of NIR-PIT effects in the pleural disseminated NSCLC mouse model, mice were randomized into 4 groups of 7 animals per group including: (1) no treatment (control); (2) only NIR light exposure at $50 \mathrm{~J} / \mathrm{cm}^{2}$ on day 1 and $100 \mathrm{~J} / \mathrm{cm}^{2}$ on day 2; (3) $100 \mu \mathrm{g}$ of tra-IR700 i.v., no NIR light exposure; (4) $100 \mu \mathrm{g}$ of tra-IR700 i.v., NIR light was administered at $50 \mathrm{~J} / \mathrm{cm}^{2}$ on day 1 after injection and $100 \mathrm{~J} / \mathrm{cm}^{2}$ on day 2 after injection. NIR light was applied transcutaneously followed by serial fluorescence imaging and BLI.

\section{Histological analysis}

To evaluate histological changes of lung at 1 day after PIT, microscopy was performed (BX51, Olympus America). Lungs with tumors were harvested and placed in $10 \%$ formalin. Serial $10-\mu m$ slice sections were fixed on glass slide for $\mathrm{H}-\mathrm{E}$ staining.

\section{Statistical Analysis}

Data are expressed as means \pm s.e.m. from a minimum of four experiments, unless otherwise indicated. Statistical analyses were carried out using a statistics program (GraphPad Prism; GraphPad Software, La Jolla, CA, USA). For multiple comparisons, a one-way analysis of variance (ANOVA) with Tukey's test was used. The cumulative probability of survival, determined herein as the tumor diameter failing to reach $2 \mathrm{~cm}$, was estimated in each group with the use 
of the Kaplan-Meier survival curve analysis, and the results were compared with the log-rank test and Wilcoxon test. $\mathrm{p}<0.05$ was considered to indicate a statistically significant difference.

\section{Results}

\section{Characterization of the cell line and the NIR-PIT effect}

To monitor optically the effect of NIR-PIT, NSCLC cell line Calu3 was genetically modified to express GFP and luciferase (Calu3-luc-GFP)(Fig. 1A). The fluorescence signals obtained with pan-IR700 and tra-IR700 using Calu3-luc-GFP cells were evaluated by FACS. After $6 \mathrm{hr}$ incubation with either pan-IR700 or tra-IR700, Calu3-luc-GFP cells showed higher brightness with tra-IR700 than with pan-IR700 consistent with the expression profile (Fig. 1B). These signals were completely blocked by the addition of excess trastuzumab, suggesting specific binding and validating that the addition of the luciferase/ GFP gene had not altered the cell expression profile. Serial fluorescence microscopy of Calu3-luc-GFP cells performed before and after NIR-PIT $\left(2 \mathrm{~J} / \mathrm{cm}^{2}\right)$ demonstrated rapidly appearing cellular swelling, bleb formation and rupture of the lysosome (Fig. 1C). Time-lapse imaging showed acute morphologic changes in the cell membrane within 25 minutes and fluorescence of PI indicating cell death (Additional File 2: Video S1). No significant changes were observed in HER2-negative 3T3 cells after exposure to NIR light, suggesting NIR-PIT induced no damage in non-target cells (Additional File 1: Fig. S1). Based on the incorporation of PI, the cell death percentage increased in a light dose dependent manner. No significant cytotoxicity was observed with NIR light exposure alone or with tra-IR700 alone (Fig. 1D). NIR-PIT was blocked with excess trastuzumab even in tra-IR700 containing media (Additional File 1: Fig. S2). Bioluminescence showed significant decreases of relative light units (RLU) in NIR-PIT treated cells (Fig. 1E). BLI also showed a decrease of luciferase activity in a light dose dependent manner (Fig. 1F). GFP fluorescence intensity was greatly reduced in dead cells (stained positive with PI), while GFP fluorescence was preserved in surviving cells (Fig. 1G). GFP fluorescence was likely reduced after NIR-PIT because the GFP was extruded from the cytoplasm after membrane rupture leading to dilution and/or denaturation. The GFP fluorescence ratio on FACS showed decreases in a light dose dependent manner, while no decrease was detected with NIR light exposure or Pan-IR700 alone (Fig. 1H). Collectively, these data suggested that the effects of NIR-PIT on Calu3-luc-GFP could be monitored with GFP fluores- cence and bioluminescence.

\section{In vivo NIR-PIT reduced tumor volume and luciferase activity in a flank xenograft model}

In vivo NIR-PIT experiments were first conducted on flank xenografts of Calu3-luc-GFP. The NIR-PIT regimen and imaging protocol are depicted in Fig. 2A. Both BLI and fluorescence decreased after NIR-PIT (Fig. 2B and Additional File 1: Fig. S3A). RLU of tumor in other groups showed a gradual increase due to tumor growth. In contrast, luciferase activity decreased 1 day after repeated NIR-PIT $\left({ }^{*} p=0.002<\right.$ 0.01, PIT vs. APC, Tukey's test with ANOVA)(Fig. $2 \mathrm{C})$. The body weight (BW) ratio showed no remarkable acute toxicity (Fig. 2D). Significant decreases $\left({ }^{* *} \mathrm{p}\right.$ $=0.0004<0.001$, PIT vs. APC, Tukey's test with ANOVA) in tumor volume were confirmed, which was consistent with luciferase activities (Fig. 2E). Survival was prolonged significantly in the PIT group $\left({ }^{* * *} \mathrm{P}<0.0001\right.$, Long-rank test and Wilcoxon test)(Fig. $2 \mathrm{~F})$. Since bioluminescence is more sensitive to tumor killing as it is based on live cells, the physical tumor volume took longer to show the effect of NIR-PIT. Collectively, these data suggest that NIR-PIT caused significant tumor reduction and prolonged survival in the in vivo flank tumor model.

\section{Characterization of the pleural disseminated NSCLC mouse model}

Prior to therapy, implanted thoracic tumors were evaluated with serial fluorescence imaging, BLI and fluorescence thoracoscopy. The implanted thoracic disseminated tumors demonstrated high activity with fluorescence imaging based on IR700, IR800 and GFP, but also high activity on bioluminescence, which co-localized with each other (Fig. 3). Fluorescence thoracoscopy indicated that disseminated tumor establishment and the good contrast of IR700 between tumors and intrathoracic organs (Fig. 3 and Additional File 3: video S2), which confirmed pleural metastases that fluoresced preferentially with tra-IR700. These data suggest that pleural disseminated NSCLC cancer mouse model with Calu3-luc-GFP cells was successfully established; intravenously injection of agent could reach the disseminated tumors.

\section{In vivo NIR-PIT effect in pleural disseminated cancer mouse model}

After treatment with NIR-PIT pleural disseminated tumors decreased in bioluminescence and fluorescence (Fig. 4A and 4B and Additional File 1: Fig. S3B). While the RLU decreased in the NIR-PIT treated tumors, RLU of tumor in other groups showed a gradual increase due to tumor growth. In contrast, luciferase activity decreased 1 day after repeated 
NIR-PIT $\left({ }^{*} p=0.0180<0.05\right.$, PIT vs. APC, Tukey's test with ANOVA)(Fig. 4C). The BW ratio showed no change (ns, PIT vs. APC, light, control, Tukey's test with ANOVA) (Fig. 4D). Taken together, these data suggest that NIR-PIT caused significant tumor reduction in vivo pleural disseminated model.

\section{In vivo NIR-PIT effect assessed with GFP fluo- rescence imaging}

Finally, to assess the effect of repeated NIR-PIT on Calu3-luc-GFP tumor in vivo, GFP fluorescence imaging was performed in both the flank model and

A

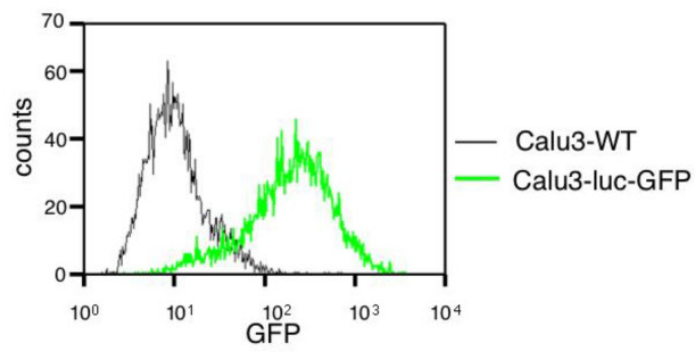

C

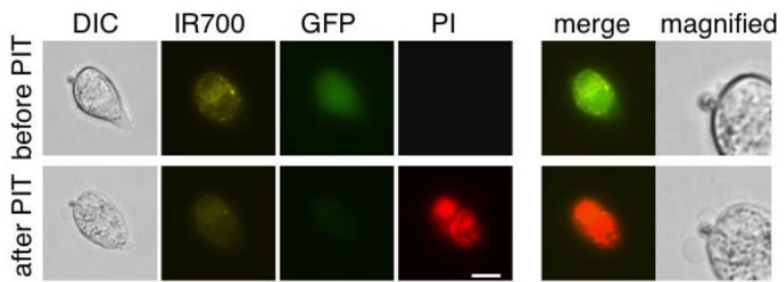

E

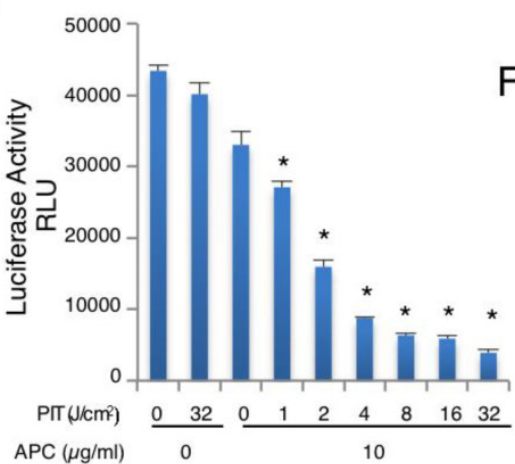

$\mathrm{F}$
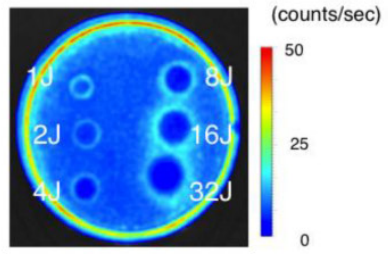

G
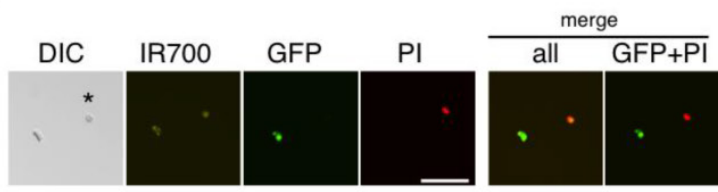

pleural disseminated model (Fig. 5A). With the flank model, both GFP/ IR700 fluorescence disappeared at 1 day after NIR-PIT, which was confirmed by ex vivo tumor imaging (Fig. 5B). Using fluorescence thoracoscopy, GFP and IR700 fluorescence disappeared (Fig. 5C and videos S3 and S4 in Additional Files 4-5). A small effusion was observed with thoracoscopy (Fig. 5C arrow). Moreover, there was no apparent damage to the normal lung by NIR-PIT as observed with histological analysis (Fig. 6).
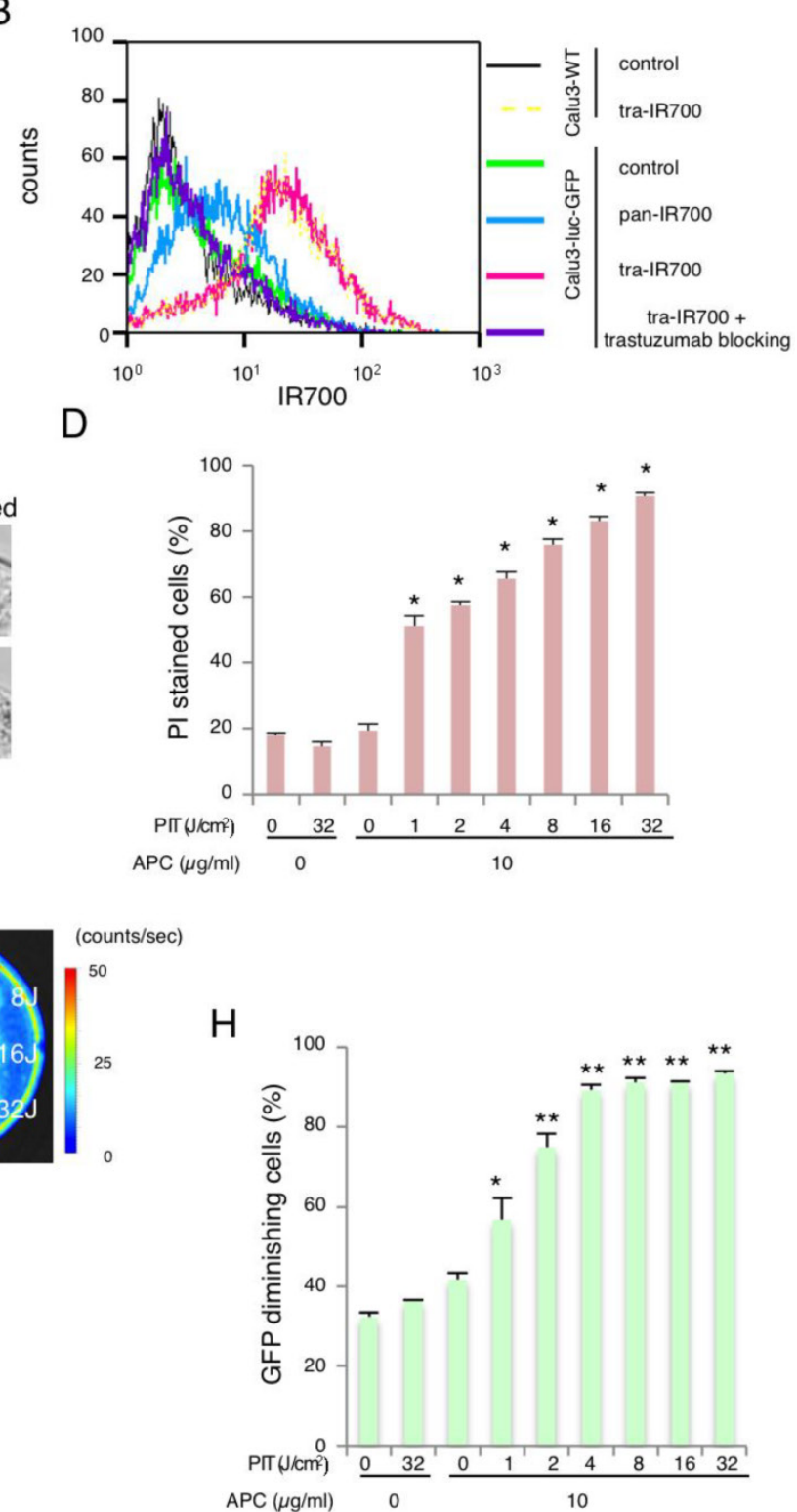

Figure 1. Characterization of cell line and evaluation of NIR-PIT effect. (A) Stable expression of GFP was confirmed with FACS. (B) Expression of HER1 and HER2 in Calu3-luc-GFP cells was examined with FACS. HER2 was overexpressed while HER1 showed normal expression. Specific binding was demonstrated with a blocking study. No remarkable change in expression of HER2 was detected compared with wild type Calu3. (C) Calu3-luc-GFP cells were incubated with tra-IR700 for 6 hr, and observed with a microscope before and after irradiation of NIR light $\left(2 \mathrm{~J} / \mathrm{cm}^{2}\right)$. Necrotic cell death was observed after exposure to NIR light $(1 \mathrm{hr}$ after NIR-PIT). Bar $=20 \mu \mathrm{m}$. (D) Membrane damage and necrosis induced by NIR-PIT was measured by dead cell count using PI staining. Cell killing increased in a NIR-light dose-dependent manner ( $\mathrm{n}=4$, ${ }^{\mathrm{P}} \mathrm{p}<0.0001$, vs. untreated control, Student's t test). (E) Luciferase activity in Calu3-luc-GFP cells was measured as relative light unit (RLU), which also decreased in a NIR-light dose-dependent manner ( $n=4,{ }^{*} p<0.0001$, vs. untreated control, Student's $t$ test). (F) Bioluminescence imaging (BLI) of a $10 \mathrm{~cm}$ dish demonstrated that luciferase activity in Calu3-luc-GFP cells decreased in a NIR-light dose-dependent manner. (G) Calu3-luc-GFP cells were incubated with tra-IR700 for $6 \mathrm{hr}$ and irradiated with NIR-light (2 J/cm²). GFP-fluorescence intensity decreased in dead cells $\left(^{*}\right)$ but was unchanged in living cells at $1 \mathrm{hr}$ after NIR-PIT. Bar $=100 \mu \mathrm{m}$. $(\mathrm{H})$ GFP fluorescence intensity decreased after NIR-PIT in a NIR-light dose-dependent manner as measured by FACS $\left(n=4,{ }^{*} p<0.005, *^{*} p<0.0001\right.$, vs. untreated control, Student's $t$ test). 


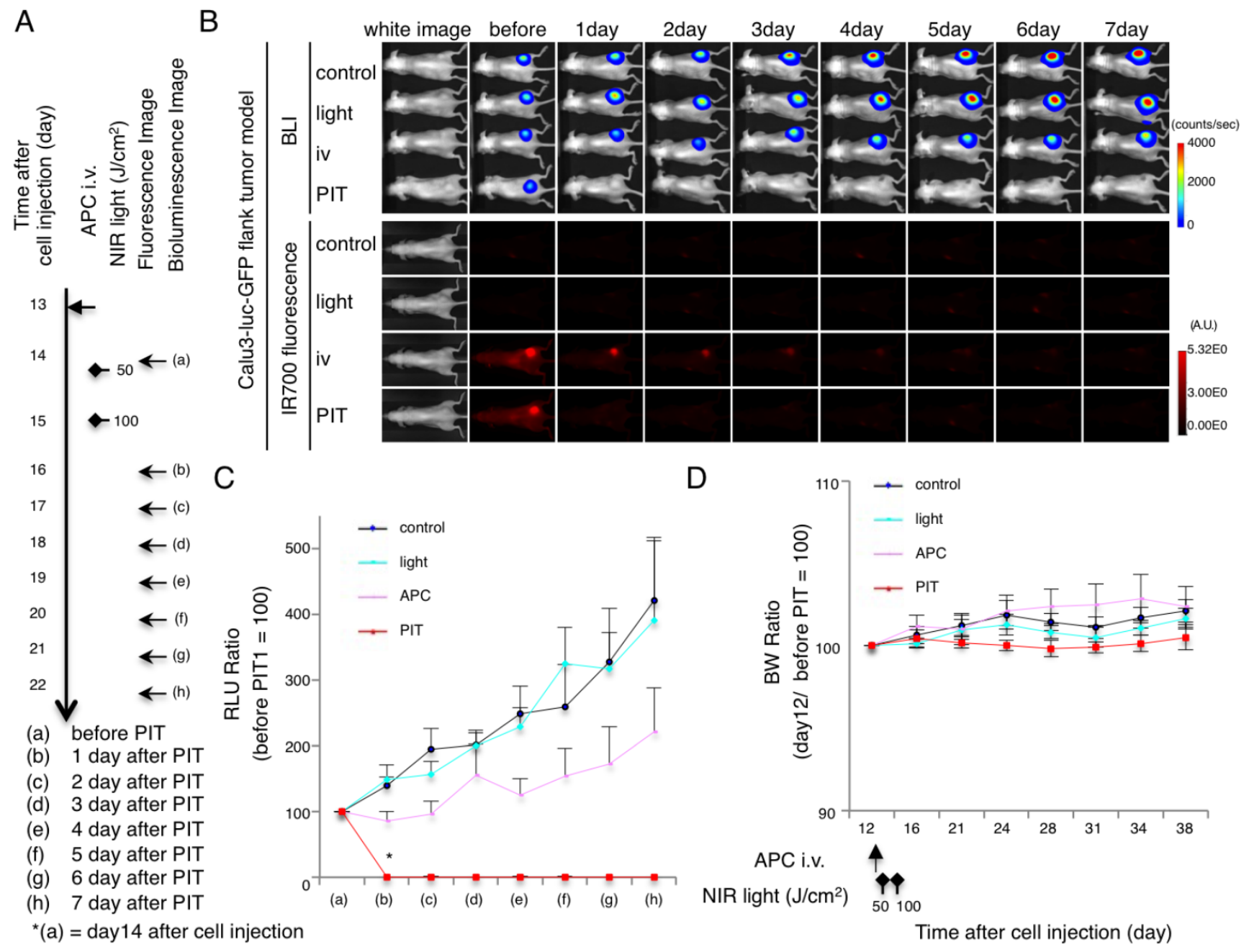

\section{$\mathrm{E}$}

APC i.v.

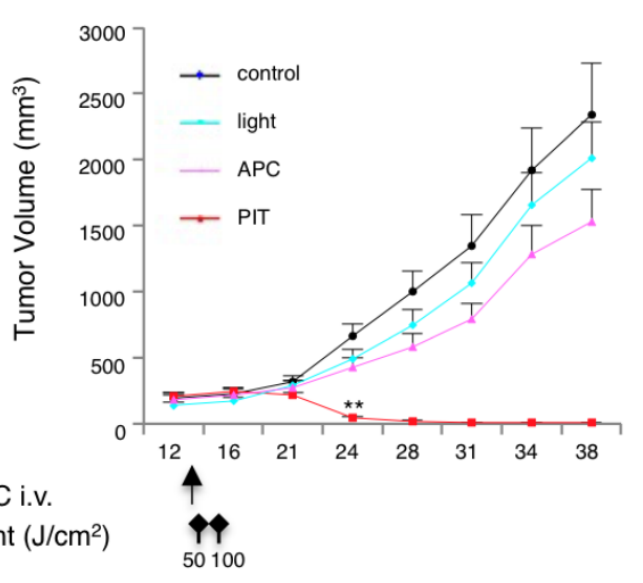

Time after cell injection (day)
$\mathrm{F}$

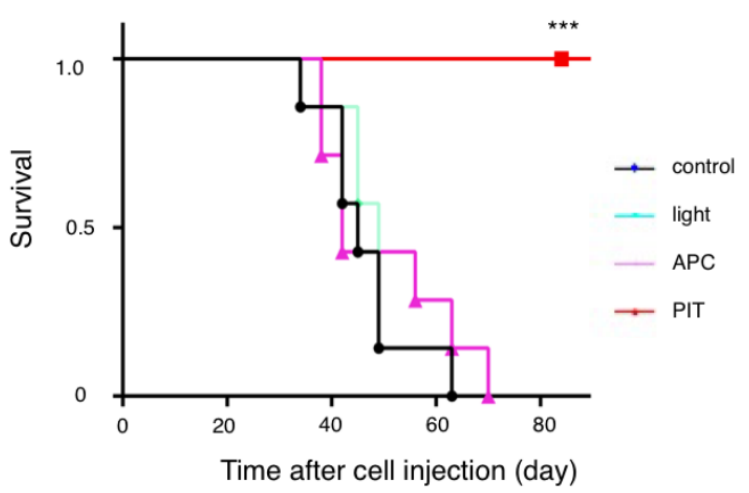

Figure 2. Evaluation of NIR-PIT in flank model. (A) The regimen of NIR-PIT is shown. Images were obtained as indicated. (B) In vivo BLI and fluorescence imaging of tumor bearing mice in response to NIR-PIT. Prior to NIR-PIT, tumors were approximately the same size and exhibited similar bioluminescence. (C) Quantitative RLU showed a significant decrease in NIR-PIT-treated tumors $(n=7$ mice in each group)(*P $=0.002<0.01$, PIT vs. APC, Tukey's test with ANOVA). (D) Body weight (BW ratio) of tumor bearing mice in response to NIR-PIT. No significant difference was detected among the groups $(n=7)$. (E) NIR-PIT leads to reductions in tumor volume ( $\mathrm{n}=10$ mice in each group) $\left({ }^{* *} \mathrm{p}=0.0004<0.001\right.$, PIT vs. APC, Tukey's test with ANOVA). Treatment is indicated below the graph. (F) Repeated NIR-PIT leads to prolonged survival in Calu3-luc-GFP tumor bearing mice ( $\mathrm{n}=7$ mice in each group) $(* * * \mathrm{P}<0.0001$, Long-rank test and Wilcoxon test). 

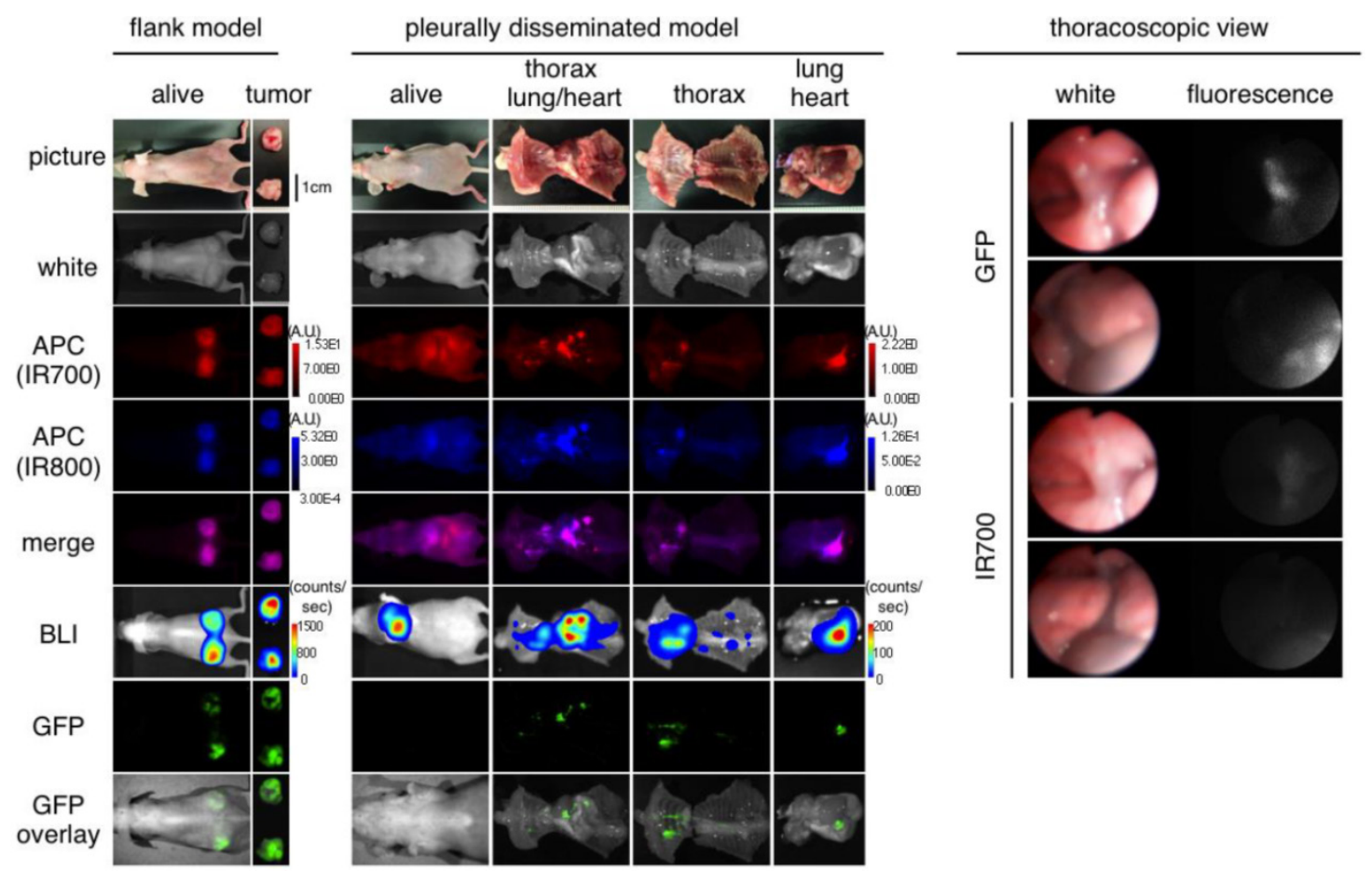

Figure 3. Characterization of the pleural disseminated NSCLC model. In vivo BLI and fluorescence (GFP/ IR700/ IR800) imaging of Calu3-luc-GFP tumor in flank and pleural disseminated model are shown and demonstrate colocalization of fluorescence. To avoid auto-fluorescence, tra-IR800 was used as well as tra-IR700 for imaging. Fluorescence thoracoscopy demonstrated that pleural metastases had both IR700 and GFP fluorescence signals. APCs were intravenously injected 1 day before the imaging.

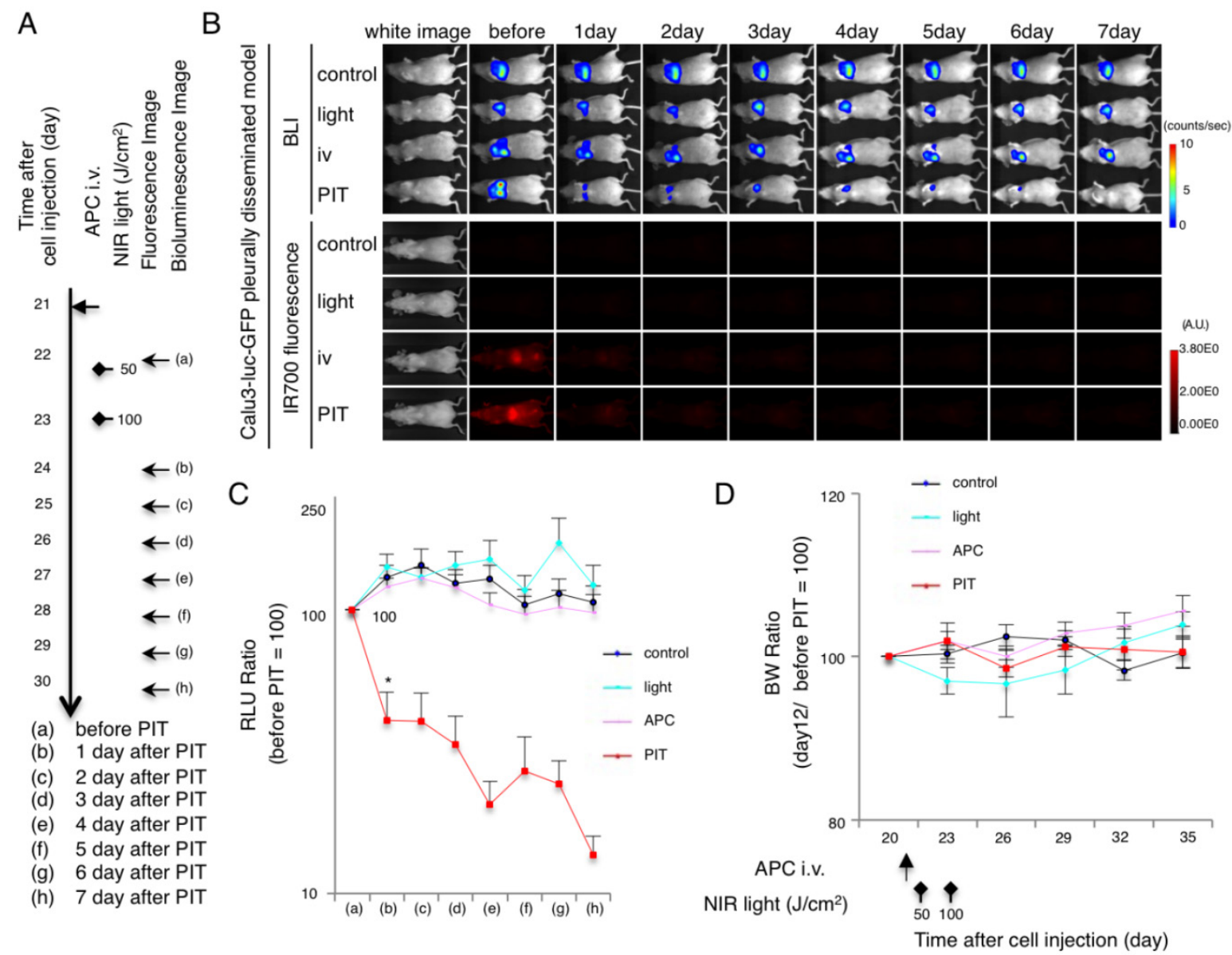

Figure 4. Evaluation of NIR-PIT effects on pleural disseminated NSCLC model by bioluminescence. (A) The regimen of NIR-PIT is shown. Images were obtained as indicated. (B) In vivo BLI and fluorescence imaging of the pleural disseminated model. Prior to treatment mice exhibiting approximately the same luciferase activity in the chest were selected. (C) Quantitative RLU in the pleural disseminated model showed a significant decrease after NIR-PIT ( $n=7$ mice in each group)(*P $=0.0180<0.05$, PIT vs. APC, Tukey's test with ANOVA). (D) BW ratio of tumor bearing mice in response to NIR-PIT demonstrates no significant difference among the groups ( $\mathrm{n}=7$ ). 
A

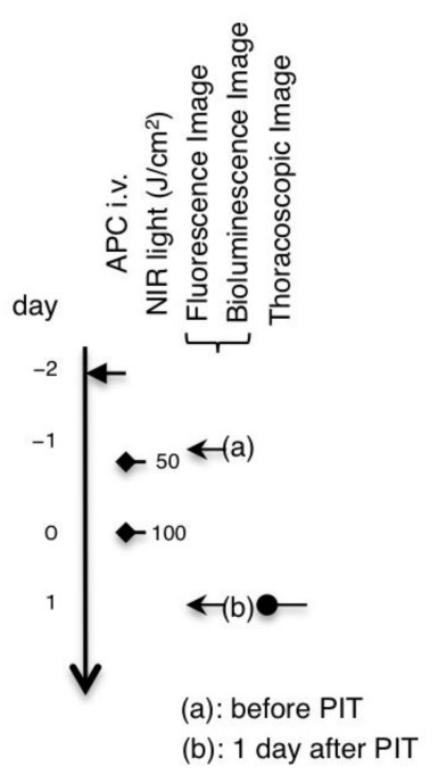

B

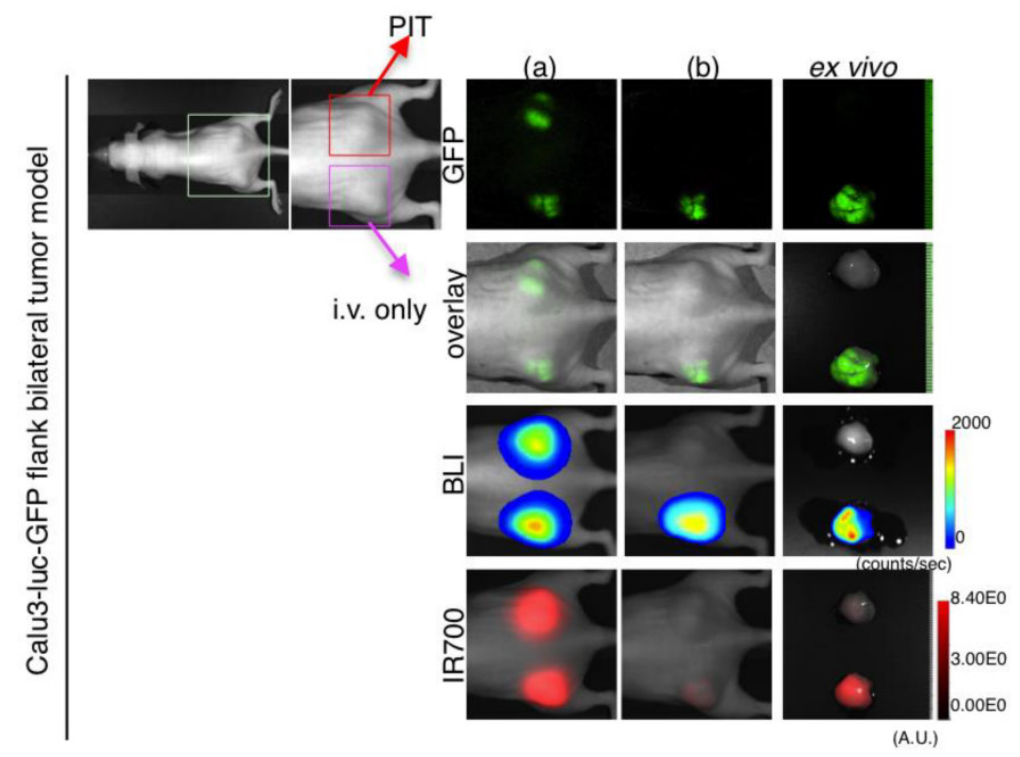

(b)

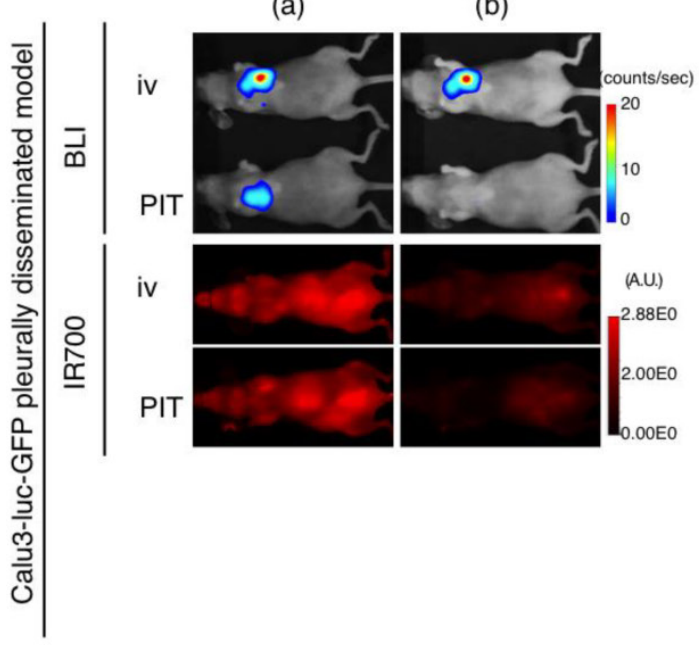

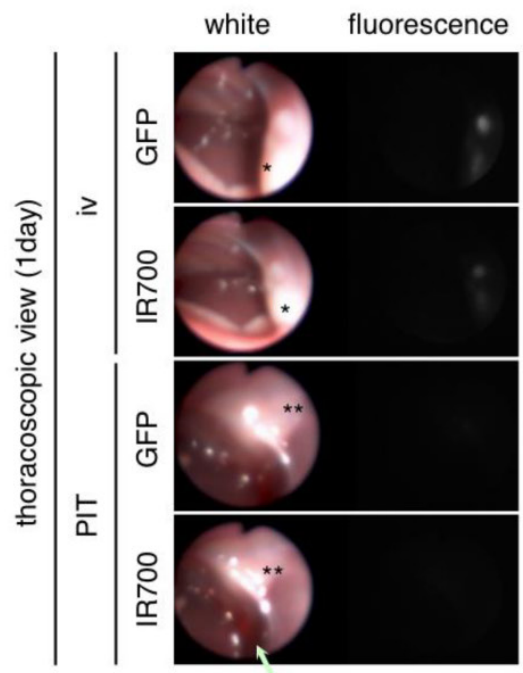

Figure 5. Evaluation of NIR-PIT effects on pleural metastassi model by GFP fluorescence imaging. (A) The regimen of NIR-PIT is shown. Images were obtained as indicated. (B) In vivo BLI and fluorescence imaging of the flank model in response to NIR-PIT. (C) In vivo BLI and thoracoscopic fluorescence imaging of pleural disseminated model in response to NIR-PIT. Both GFP-fluorescence and IR700 fluorescence intensity decreased in Calu3-luc-GFP disseminated tumors (*) after NIR-PIT. A small reactive pleural effusion was observed (arrow).
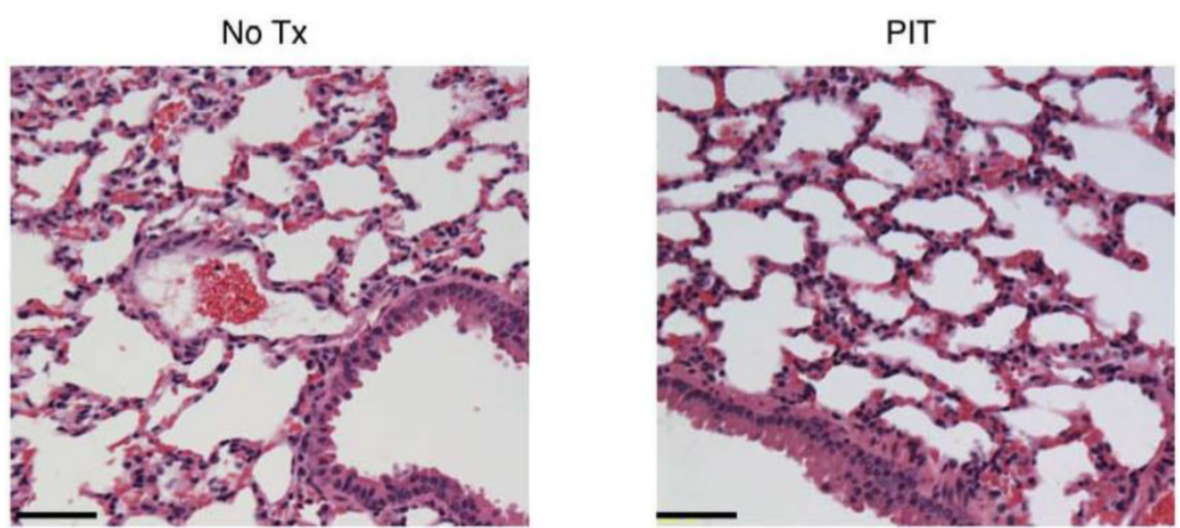

Figure 6. Histological evaluation of NIR-PIT effect on lung. No apparent damage to the lung was showed by HE-staining at 1 day after NIR-PIT compared to no Tx. Bar $=50 \mu \mathrm{m}$. 


\section{Discussion}

In this study, we demonstrate that an APC can be delivered to both flank and intrathoracic tumor after intravenous injection and that subsequent NIR-PIT can be successfully performed transcutaneously to the mouse thorax with acceptable morbidity. Among the imaging tools used to document tumor regression, which included BLI, fluorescence imaging and fluorescence thoracoscopy, the latter two could be used in clinical practice using the IR700 dye $[19,20]$. BLI using firefly luciferase, although less suitable for clinical translation, was useful as a primary outcome measure as it requires both oxygen and ATP to actively transport the substrate luciferin and subsequently catalyze the photochemical reaction [21,22]. Since NIR-PIT-induced necrotic cell death releases ATP, BLI is an appropriate and sensitive biomarker for NIR-PIT $[16,23]$. In vivo GFP fluorescence imaging enables the full process of tumorigenesis, treatment, regression, metastasis, or recurrence, to be detected although this also is not translatable [24]. The high level of GFP tumor fluorescence in this model permitted imaging with quantification of tumor growth and dissemination without the need for additional contrast agents. By employing cytoplasmic GFP expressing cells, antitumor effects induced by NIR-PIT could be clearly monitored as extrusion of GFP from treated cells resulted in a diminution of signal [15]. The development of a mini-endoscope mimicking thoracoscopy permits intrathoracic fluorescence imaging and is the most likely method by which NIR-PIT would be administered and monitored in humans. By changing the filter sets, multicolor endoscopic imaging becomes possible to simultaneously monitor tumor regression with GFP fluorescence and accumulation of APC with IR700 fluorescence as shown in videos S3 and S4 [25] (Additional Files 4-5). Both real-time color capability and direct access to the disseminated tumors, resulted in much higher resolution imaging. From the photophysical point of view, the endoscope can minimize light scattering and absorbance that is caused by overlapping tissue, resulting in more precise depiction of the lesion.

In this study, we use a pleural disseminated tumor model by simple tumor cell injection in the thoracic cavity. A variety of animal models could be used including percutaneous orthotopic injection (POI), surgical orthotopic injection (SOI), and transpleural orthotopic injection (TOI)[26]. Various advantages and disadvantages exist among these models. For example, SOI requires high skills and is invasive, resulting in high pre-procedure mortality, however SOI is thought to be more physiologic than others $[26,27]$. The POI model has the advantages of sim- plicity and less invasiveness with a very low pre-procedure mortality rate [26]. A recent study reported that implantation rates were similar among these models [26]. With these considerations, we chose the POI approach. This approach had a high implantation rate (around 85\%) of NSCLC pleural dissemination, which was confirmed by non-invasive BLI.

The survival of patients with NSCLC patients with pleural disseminations is only 6 to 9 months even with systemic chemotherapy [3]. Surgery is not currently performed because of its morbidity and limited benefit [28]. While not likely to be curative, NIR-PIT could offer the benefit of local control with minimal invasiveness. Moreover, NIR-PIT could be readily used as an adjunct to conventional surgery at the time of initial diagnosis.

There are several limitations to this study. First, not all lung cancers overexpress HER2, and therefore this particular target may not be ideal in other lung cancers. Fortunately, NIR-PIT has proven effective with almost all APCs with which it has been attempted and therefore, it is likely that the proper APC or combination of APCs could be found to treat a specific phenotype of lung cancer cell membrane expression [13-15,29,30]. We were also unable to determine the long-term side effects of NIR-PIT in this limited model. Short-term studies of the mice demonstrated no apparent adverse events after NIR-PIT. It is possible that sudden widespread cell necrosis could cause either acute or delayed toxicity but none was observed in this model. Only small reactive pleural effusions were observed by thoracoscopy. Additionally, it is clear that NIR-PIT alone will not be sufficient to cure thoracic metastases, although the use of NIR light to activate IR700 will produce deeper tissue penetration within larger masses than the shorter wavelengths of light used in conventional PDT photoactivation or other light therapies such as those using UV light [31]. Therefore, we foresee NIR-PIT as an adjuvant to surgery with an initial debulking procedure followed by NIR-PIT to "mop up" residual disease. Furthermore, it is interesting to consider the possibility that systemic chemotherapy may be more effective after NIR-PIT. Previous studies have shown that NIR-PIT causes treated tumors to exhibit increased permeability to nano-sized drugs. Therefore, current or future chemotherapies for lung cancer may benefit from prior treatment with NIR-PIT [32]. Finally, in this study we irradiated transcutaneously which is difficult to translate to the clinic, however, it would be feasible to deliver light via thoracoscopy, bronchoscopy or even during open-surgery. Thus, although this particular animal model is not directly translatable, the principle of 
treating thoracic malignancies with light therapy is feasible.

In conclusion, this study demonstrates that NIR-PIT effectively treated pleural metastases in a mouse model of NSCLC. NIR-PIT could be a promising adjuvant for treating pleural carcinomatosis replacing or adding to existing therapies such as surgery and chemotherapy.

\section{Supplementary Material}

Additional File 1:

Supplementary Figures S1-S3.

http://www.thno.org/v05p0698s1.pdf

Additional File 2:

Video S1.

http://www.thno.org/v05p0698s2.mp4

Additional File 3:

Video S2.

http:/ / www.thno.org/v05p0698s3.mp4

Additional File 4:

Video S3.

http:/ / www.thno.org/v05p0698s4.mp4

Additional File 5:

Video S4.

http://www.thno.org/v05p0698s5.mp4

\section{Acknowledgements}

This research was supported by the Intramural Research Program of the National Institutes of Health, National Cancer Institute, Center for Cancer Research. K.S. is supported with JSPS Research Fellowship for Japanese Biomedical and Behavioral Researchers at NIH.

\section{Contributions}

K.S. mainly conducted experiments, performed analysis and wrote the manuscript; T.N. conducted thoracoscopy with K.S. P.L.C. wrote the manuscript and supervised the project; and H.K. planned and initiated the project, designed and conducted experiments, wrote the manuscript, and supervised the entire project.

\section{Competing Interests}

The authors have declared that no competing interest exists.

\section{References}

1 Siegel R, Ma J, Zou Z, Jemal A. Cancer statistics, 2014. CA cancer J Clin 2014;64:9-29. doi:10.3322/caac.21208.

2 Powell C a, Halmos B, Nana-Sinkam SP. Update in lung cancer and mesothelioma 2012. Am J Respir Crit Care Med 2013;188:157-66. doi:10.1164/rccm.201304-0716UP.

3 Reyes L, Parvez Z, Regal AM TH. Neoadjuvant chemotherapy and operations in the treatment of lung cancer with pleural effusion. J Thorac Cardiovasc Surg 1991:101:946-7.

4 Goldstraw P, Crowley J. The IASLC Lung Cancer Staging Project: proposals for the revision of the TNM stage groupings in the forthcoming (seventh) edition of the TNM Classification of. J Thorac Oncol 2007;2:706-14.
5 Pass H, DeLaney T, Tochner Z. Intrapleural photodynamic therapy: results of a phase I trial. Ann Surg Oncol 1994;1:28-37.

6 Pass HI, Temeck BK, Kranda K, Thomas G, Russo A, Smith P, et al. Phase III randomized trial of surgery with or without intraoperative photodynamic therapy and postoperative immunochemotherapy for malignant pleural mesothelioma. Ann Surg Oncol 1997;4:628-33. doi:10.1007/BF02303746.

7 Friedberg JS, Mick R, Stevenson JP, Zhu T, Busch TM, Shin D, et al. Phase II trial of pleural photodynamic therapy and surgery for patients with non-small-cell lung cancer with pleural spread. J Clin Oncol 2004;22:2192-201. doi:10.1200/JCO.2004.07.097.

8 Mew D, Wat C, Towers GHN, Levy JG. PHOTOIMMUNOTHERAPY: TREATMENT OF ANIMAL TUMORS WITH TUMOR-SPECIFIC. I Immunol 1983;130:1473-7.

9 Oseroff a R, Ohuoha D, Hasan T, Bommer JC. Antibody-targeted photolysis: selective photodestruction of human T-cell leukemia cells using monoclonal antibody-chlorin e6 conjugates. Proc Natl Acad Sci U S A 1986;83:8744-8.

10 Goff BA, Hermanto U, Rumbaugh J, Blake J, Bamberg M, Hasan T. Photoimmunotherapy and biodistribution with an OC125-chlorin immunoconjugate in an in vivo murine ovarian cancer model. $\mathrm{Br} J$ Cancer 1994;70:474-80.

11 Vrouenraets MB, Visser GWM, Stewart FA, Stigter M, Oppelaar H, Postmus PE, et al. Development of meta -Tetrahydroxyphenylchlorin-Monoclonal Antibody Conjugates for Photoimmunotherapy. Cancer Reserch 1999;59:1505-13.

12 Vrouenraets MB, Visser GWM, Stigter M, Oppelaar H, Snow GB, van Dongen GA. Targeting of aluminum (III) phthalocyanine tetrasulfonate by use of internalizing monoclonal antibodies: Improved efficacy in photodynamic therapy. Cancer Res 2001;61:1970-5.

13 Mitsunaga M, Ogawa M, Kosaka N, Rosenblum LT, Choyke PL, Kobayashi H. Cancer cell - selective in vivo near infrared photoimmunotherapy targeting specific membrane molecules. Nat Med 2011;17:1685-91. doi:10.1038/nm.2554.

14 Sato K, Watanabe R, Hanaoka H, Harada T, Nakajima T, Kim I, et al. Photoimmunotherapy: Comparative effectiveness of two monoclonal antibodies targeting the epidermal growth factor receptor. Mol Oncol 2014;8:620-32. doi:10.1016/j.molonc.2014.01.006.

15 Sato K, Choyke PL, Kobayashi H. Photoimmunotherapy of Gastric Cancer Peritoneal Carcinomatosis in a Mouse Model. PLoS One 2014;9:e113276. doi:10.1371/journal.pone.0113276.

16 Sato K, Hanaoka H, Watanabe R, Nakajima T, Choyke PL, Kobayashi H. Near infrared photoimmunotherapy in the treatment of disseminated peritoneal ovarian cancer. Mol Cancer Ther 2015 Jan;14(1):141-50. doi: 10.1158/1535-7163.MCT-14-0658. Epub 2014 Nov 21.

17 Sato K, Watanabe T, Wang S, Kakeno M, Matsuzawa K, Matsui T, et al. Numb controls E-cadherin endocytosis through p120 catenin with aPKC. Mol Biol Cell 2011;22:3103-19. doi:10.1091/mbc.E11-03-0274.

18 Mitsunaga M, Nakajima T, Sano K, Choyke PL, Kobayashi H. Near-infrared Theranostic Photoimmunotherapy (PIT): Repeated Exposure of Light Enhances the Effect of Immunoconjugate. Bioconjug Chem 2012;23:604-9. doi:10.1021/bc200648m.

19 Conway JRW, Carragher NO, Timpson P. Developments in preclinical cancer imaging: innovating the discovery of therapeutics. Nat Rev Cancer 2014:14:314-28, doi:10.1038/nrc3724.

20 De Jong M, Essers J, van Weerden WM. Imaging preclinical tumour models: improving translational power. Nat Rev Cancer 2014;14:481-93. doi:10.1038/nrc3751.

21 Rehemtulla A, Stegman LD, Cardozo SJ, Gupta S, Hall DE, Contag CH, et al. Rapid and Quantitative Assessment of Cancer Treatment Response Using In Vivo Bioluminescence Imaging 1 Days post sham treatment. Neoplasia 2000:2:491-5

22 Contag PR, Olomu IN, Stevenson DK CC. Bioluminescent indicators in living mammals. Nat Med 1998;4:245-7.

23 Mitsunaga M, Nakajima T, Sano K, Kramer-Marek G, Choyke PL, Kobayashi $\mathrm{H}$. Immediate in vivo target-specific cancer cell death after near infrared $\begin{array}{lll}\text { photoimmunotherapy. } & \text { BMC } & \text { Cancer. }\end{array}$ doi:10.1186/1471-2407-12-345.

24 Hoffman RM. The multiple uses of fluorescent proteins to visualize cancer in vivo. Nat Rev Cancer 2005;5:796-806. doi:10.1038/nrc1717.

25 Mitsunaga M, Kosaka N, Kines RC, Roberts JN, Lowy DR, Schiller JT, et al. In vivo longitudinal imaging of experimental human papillomavirus infection in mice with a multicolor fluorescence mini-endoscopy system. Cancer Prev Res (Phila) 2011;4:767-73. doi:10.1158/1940-6207.CAPR-10-0334.

26 Mordant $\mathrm{P}$, Loriot $\mathrm{Y}$, Lahon $\mathrm{B}$, Castier $\mathrm{Y}$, Leseche G, Soria JC, et al. Bioluminescent orthotopic mouse models of human localized Non-Small cell lung cancer: Feasibility and identification of circulating tumour cells. PLoS One 2011;6:10-2. doi:10.1371/journal.pone.0026073.

27 Yang $M$, Hasegawa S, Jiang $\mathrm{P}$, Wang $\mathrm{X}$, Tan $\mathrm{Y}$, Chishima $\mathrm{T}$, et al. Widespread skeletal metastatic potential of human lung cancer revealed by green fluorescent protein expression. Cancer Res 1998;58:4217-21.

28 Yokoi K, Matsuguma H, Anraku M. Extrapleural pneumonectomy for lung cancer with carcinomatous pleuritis. J Thorac Cardiovasc Surg 2002;123:184-5. doi:10.1067/mtc.2002.118039.

29 Shirasu N, Yamada H, Shibaguchi H, Kuroki M, Kuroki M. Potent and specific antitumor effect of CEA-targeted photoimmunotherapy. Int I Cancer 2014;135:2697-710. doi:10.1002/ijc.28907. 
30 Nakajima T, Sano K, Choyke PL, Kobayashi H. Improving the efficacy of Photoimmunotherapy (PIT) using a cocktail of antibody conjugates in a multiple antigen tumor model. Theranostics 2013;3:357-65. doi: $10.7150 /$ thno. 5908

31 Hiroshima Y, Maawy A, Zhang Y, Sato S, Murakami T,Yamamoto M, et al. Fluorescence-guided surgery in combination with UVC irradiation cures metastatic human pancreatic cancer in orthotopic mouse models. PLoS One 2014;9:e99977. doi:10.1371/journal.pone.0099977.

32 Sano K, Nakajima T, Choyke PL, Kobayashi H. Markedly Enhanced Permeability and Retention Effects Induced by Photo-immunotherapy of Tumors. ACS Nano 2013;7:717-24. doi:10.1021/nn305011p. 